\title{
Limitantes da evolução ponderal de mulheres com excesso de peso após intervenção nutricional
}

\author{
Limiting of unsatisfactory weight loss among overweight women \\ after nutritional intervention
}

\begin{abstract}
Mariana Souza Lopes*
Maria Júlia Gonçalves Barbosa**

Nathália Luíza Ferreira*

Luana Caroline dos Santos*

Aline Cristine Souza Lopes*

Resumo

Insuficiente redução ponderal em intervenções conduzidas com indivíduos com excesso de peso remete à necessidade de constatar os aspectos preditores do insucesso, visando redirecionar ações, contribuindo para seu maior êxito. Assim, objetivou-se identificar os fatores preditores da evolução ponderal insatisfatória de mulheres com excesso de peso após acompanhamento nutricional na Atenção Primária à Saúde. Estudo retrospectivo realizado com mulheres com excesso de peso após 12 meses de acompanhamento nutricional individual mensal. Dados coletados por anamnese constando de dados sociodemográficos, morbidades referidas, hábitos e consumo alimentar, além de medidas antropométricas. Classificou-se a evolução ponderal como insatisfatória (<5\% do peso inicial) e satisfatória ( $>=5 \%)$, sendo os grupos comparados a partir dos testes estatísticos t de Student, Mann Whitney, Qui-quadrado e Exato de Fisher $(p<0,05)$.

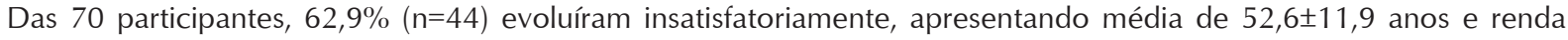
per capita mensal de $63,6 \%$ do salário mínimo vigente $(18,6 \% ; 198,3 \%)$, características semelhantes às das mulheres com evolução satisfatória. Usuárias com evolução insatisfatória apresentaram, no início do acompanhamento, maior prevalência de hipertensão arterial, dormiam menos horas por noite, assistiam televisão e/ou utilizavam computador por mais tempo e relataram menor sudorese durante a prática de atividade física. Os resultados apontam que o insucesso da redução ponderal pode estar relacionado a aspectos que extrapolam a adoção de uma alimentação adequada, perpassando por fatores como sono, sedentarismo e presença de comorbidades. Sugere-se assim, o desenvolvimento de intervenções diferenciadas e ampliadas quanto à abordagem terapêutica, que considerem a complexidade envolvida no manejo do excesso de peso, almejando o maior sucesso das ações.
\end{abstract}

Palavras-chave: Aconselhamento. Atenção Primária à Saúde. Obesidade. Perda de Peso. Sobrepeso.

\begin{abstract}
Insufficient weight loss in interventions conducted with overweight individuals demonstrate the need to identify their predictors in order to redirect actions and promote greater success. Thus, we aimed to identify predictors of unsatisfactory weight loss among overweight women after nutritional monitoring in Primary Health Care. This was a retrospective study of overweight women after 12 months of monthly individual nutritional monitoring. Data were collected through anamnesis containing sociodemographic data, reported morbidities, habits and food consumption, as well as anthropometric measurements. The weight loss was classified as unsatisfactory $(<5 \%$ of initial weight $)$ and satisfactory $(\geq 5 \%)$, and these groups were compared using the Student's t-test, Mann Whitney, Chi-square and Fisher's Exact statistical tests. $(\mathrm{p}<0.05) .62 .9 \%$ of the 70 participants $(n=44)$ presented unsatisfactory weight loss. They were aged $52.6 \pm 11.9$ years old who on average had a monthly per capita income of $63.6 \%$ of minimum salary $(18.6 \%$; $198.3 \%)$; characteristics similar to those women who progressed satisfactorily. Those with unsatisfactory weight loss presented a greater prevalence of hypertension, slept less hours per night, watched TV or used computer for longer periods of time, and reported less sweating during physical activity at the start of monitoring. The results show that the failure to lose weight may be related to aspects that go beyond the adoption of adequate food, including factors such as sleep, physical inactivity, and comorbidities. It is therefore suggested that the development of differentiated and expanded interventions regarding the therapeutic approach, consider the complexity involved in instituting more effective, healthy, and satisfying weight-loss programs
\end{abstract}

Keywords: Counseling. Obesity. Overweight. Primary Health Care. Weight Loss.

DOI: $10.15343 / 0104-7809.20164004433443$

\footnotetext{
*Universidade Federal de Minas Gerais, Belo Horizonte, Minas Gerais, Brasil.

** Prefeitura Municipal de Florianópolis, Secretaria da Saúde. Florianópolis, SC, Brasil.

Os autores declaram não haver conflito de interesses.
} 


\section{INTRODUÇÃO}

O excesso de peso possui etiologia multifatorial e relação direta com o desenvolvimento de doenças crônicas não transmissíveis (DCNT), como diabetes mellitus (DM), hipertensão arterial (HA), dislipidemias e doenças cardiovasculares. Por conseguinte, apresenta relevante impacto nos custos em saúde ${ }^{1}$, o que reitera a necessidade de intervenções abrangentes com vistas à sua prevenção e controle ${ }^{1,2}$.

A redução superior a 5\% do peso corporal tem sido apontada como indicativo de efetividade de intervenções por promover melhorias significativas na saúde dos indivíduos ${ }^{3,4}$, oportunizando diversos benefícios, como decréscimo dos valores de pressão arterial, glicemia de jejum, colesterol total e frações aterogênicas ${ }^{5}$. Entretanto, de modo geral, as intervenções nutricionais têm obtido altas taxas de evolução ponderal insatisfatória, tornando, por vezes, frustrante o tratamento do excesso de peso, tanto para os profissionais quanto para os pacientes ${ }^{6,7}$.

\section{MATERIAL E MÉTODOS}

\section{Tipo e população de estudo}

Trata-se de estudo retrospectivo, derivado de estudo de intervenção conduzido com mulheres com excesso de peso que participaram de 12 meses de acompanhamento nutricional individual mensal em duas Unidades Básicas de Saúde (UBS) de uma capital da região sudeste do País.

As participantes com excesso de peso eram encaminhadas por profissionais do Programa Academia da Saúde, serviço público de promoção da saúde do município, para o acompanhamento nutricional nas UBS, onde o estudo foi realizado.

Os critérios de inclusão deste estudo foram: ser mulher, adulta ou idosa (20 anos ou mais de idade), apresentar excesso de peso (IMC $\geq 25,0$ $\mathrm{kg} / \mathrm{m}^{2}$ entre adultas e $\mathrm{IMC}>27,0 \mathrm{~kg} / \mathrm{m}^{2}$ entre idosas) e ter completado os 12 meses de
Fatores como sexo, idade, elevados valores de Índice de Massa Corporal $(\mathrm{IMC})^{8}$ e controle das comorbidades ${ }^{9}$ têm sido apontados como relevantes barreiras para o sucesso das intervenções, assim como a ausência de apoio familiar, falta de motivação e resultados negativos em tentativas pregressas de redução do peso $^{4,5}$. Outra questão que pode interferir no alcance das metas de redução do peso é o tempo de acompanhamento e intensidade das intervenções ${ }^{10}$.

A identificação de fatores preditores da insatisfatória evolução ponderal de indivíduos com excesso de peso pode contribuir para o desenvolvimento adequado das ações, de acordo com as características dos sujeitos, contribuindo para o seu êxito. Neste contexto, o presente estudo se propõe a investigar os fatores preditores da evolução ponderal insatisfatória (redução de peso inferior a 5\% do peso corporal) de mulheres com excesso de peso após acompanhamento nutricional na Atenção Primária à Saúde (APS).

acompanhamento nutricional nas UBS.

\section{Coleta de dados}

Os dados foram coletados entre 2007 e 2009 por acadêmicos de Nutrição e pós-graduandos devidamente capacitados. As usuárias foram avaliadas na linha de base e reavaliadas após 12 meses de intervenção nutricional individual.

Para controle da fidedignidade e qualidade das informações adotou-se um manual, elaborado pela equipe de pesquisa, para orientar a coleta de dados, cujo conteúdo foi trabalhado periodicamente com os entrevistadores em treinamentos regulares. Todos os entrevistadores foram supervisionados pelos pesquisadores responsáveis.

Os equipamentos utilizados, balança digital Marte ${ }^{\circledR}$, modelo PP2005 LC200-PP, com capacidade máxima de 199,95kg e sensibilidade 
de $50 \mathrm{~g}$ e estadiômetro portátil Alturaexata $^{\circledR}$, com capacidade máxima de 2,13m, foram certificados por órgão competente, calibrados e vistoriados.

Análises de consistência das anamneses e do banco de dados foram continuamente realizadas.

As variáveis coletadas constaram de características sociodemográficas (idade, ocupação profissional, escolaridade e renda per capita mensal) e de saúde (morbidade referida; uso de medicamentos; relato de prática regular de exercício físico - no mínimo três vezes por semana, durante 60 minutos, e presença de sudorese durante a prática; hábitos sedentários e de sono; autopercepção corporal e de saúde; satisfação corporal e tentativa de emagrecimento nos últimos seis meses); hábitos alimentares (número de refeições por dia, realização do desjejum, ingestão de líquidos durante as refeições principais, consumo per capita de sal, óleo e açúcar e modo de preparo de alimentos - cru, cozido, refogado e frito); frequência alimentar verificada pelo Questionário de Frequência Alimentar (QFA) qualitativo $^{11}$ e por pergunta breve sobre o consumo de frutas no último mês.

O QFA qualitativo, referente aos últimos seis meses, contemplou uma lista de 28 alimentos, e foi revisado a partir de análise de Recordatório Alimentar de 24 horas realizado nos serviços de saúde em estudo ${ }^{11}$. O QFA foi constituído por cinco categorias de frequência de consumo: diário, semanal, mensal, raro e nunca, as quais foram recategorizadas para fins de análise em "Consumo diário" e "Outros", que abarcou a ingestão semanal, mensal, rara ou nunca. Para o consumo de frutas utilizou-se a categorização de " $\geq 4$ vezes por semana" $\mathrm{e}$ " $<4$ vezes por semana".

Adicionalmente, foi realizada avaliação antropométrica, incluindo a aferição do peso corporal, estatura, circunferências da cintura (CC) e do quadril (CQ). As medidas de peso e estatura permitiram calcular o IMC, sendo este classificado diferentemente para mulheres adultas $^{12}$ e idosas ${ }^{13}$. A partir dos valores de CC e CQ calculou-se a Razão cintura-quadril (RCQ). Valores de CC $\geq 80,0 \mathrm{~cm}$ e $\geq 88,0$ $\mathrm{cm}$ foram considerados como risco elevado e muito elevado de complicações metabólicas associadas à obesidade, respectivamente, ao passo que para RCQ, valores acima de 0,85 foram classificados como risco para o desenvolvimento de doenças ${ }^{14}$.

\section{Acompanhamento Nutricional}

O estudo de intervenção do qual derivou este trabalho consistiu na realização de aconselhamento nutricional centrado nas necessidades do indivíduo ${ }^{2}$ e pautado na Educação Alimentar e Nutricional. Os temas relacionados à alimentação e nutrição tratados durante os atendimentos basearamse em evidências científicas e publicações do Ministério da Saúde do Brasil, como os Cadernos de Atenção Básica e a primeira edição do Guia Alimentar para a População Brasileira, vigente à época do estudo.

Materiais impressos elaborados pela equipe de pesquisa, contendo as principais metas do tratamento, sendo preferencialmente ilustrativos e com linguagem simples, foram utilizados. As orientações consideraram o contexto biopsicossocial das participantes, estimulando sua autonomia para o autocuidado e a realização de mudanças gradativas e duradouras para a melhoria da saúde ${ }^{15}$.

\section{Análise dos dados}

A variável de desfecho consistiu na evolução insatisfatória do peso corporal, definida pela redução ponderal inferior a $5 \%$ do peso inicial após 12 meses de acompanhamento nutricional, calculada a partir do delta percentual da evolução do peso (Figura 1). As variáveis explicativas, obtidas na linha de base, incluíram os dados sociodemográficos, de saúde, hábitos e consumo alimentar, além de medidas antropométricas.

As análises estatísticas foram realizadas com o auxílio dos programas Statistical Package for the Social Sciences - SPSS (versão 17.0, SPSS inc., Chicago, 2008) e Stata (versão 13.0).

Aplicou-se o teste Shapiro-Wilk para verificar a normalidade das variáveis numéricas contínuas. Para as variáveis com distribuição paramétrica, os dados foram apresentados como média e desvio padrão, e as demais variáveis como medianas e valores mínimo e 
máximo.

Para a comparação dos dados da linha de base das usuárias que evoluíram insatisfatoriamente com aquelas que atingiram a redução de pelo menos $5 \%$ do peso inicial foram aplicados os testes estatísticos $t$ de Student, Mann Whitney, Qui-quadrado e Exato de Fisher, adotando-se o nível de significância de 5\%.

DELTA \% evolução ponderal $=\{[($ Peso após 12 meses de acompanhamento $(\mathrm{kg})$ Peso inicial (Kg) ) $\div$ Peso inicial (kg)] * 100\}

Figura 1 - Equação para cálculo de delta percentual de redução ponderal. Belo Horizonte, Brasil. 2007-2009.

\section{Aspectos Éticos}

Este estudo foi aprovado pelos Comitês de Ética da Universidade (ETIC 328/06) e da Prefeitura Municipal (nº: 017/2007).
Todas as participantes foram informadas sobre a pesquisa e assinaram o Termo de Consentimento Livre e Esclarecido, conforme Resolução 196/96 do Conselho Nacional de Saúde.

\section{RESULTADOS}

Setenta mulheres atenderam aos critérios de inclusão do estudo. Destas 62,9\% ( $n=44)$ apresentaram evolução ponderal insatisfatória, sendo seu estado nutricional no início do acompanhamento semelhante ao grupo de mulheres que evoluíram satisfatoriamente. Elevadas prevalências de risco de complicações metabólicas associadas à obesidade e de desenvolvimento de doenças cardiovasculares também foram identificadas, mas também sem diferenças entre os grupos na linha de base (Tabela 1).

Embora não tenham sido observadas diferenças estaticamente significantes, inadequações alimentares na linha de base foram bastante prevalentes, com destaque para o consumo infrequente de frutas, legumes, verduras e leite; a frequente ingestão de frituras; além do modo de preparo menos saudável (frito ou refogado) das carnes, couve e ovos (Tabela 2).

Para o total das usuárias entrevistadas $(n=70)$, independente da evolução ponderal, observouse média de 52 anos de idade, escolaridade em torno de cinco anos de estudo e renda per capita mensal de aproximadamente $\mathrm{R} \$ 267,00$, cerca de $63,6 \%$ da média do salário mínimo vigente no período da pesquisa (Tabela 3 ), sem diferenças significativas.

Dentre as variáveis da linha de base investigadas, se associaram à evolução ponderal insatisfatória a presença de HA $(60,5 \%$ vs. $34,6 \%$ entre as que evoluíram satisfatoriamente; $\mathrm{p}=0,037$ ), o hábito de dormir menos horas por noite $(6,6 \pm 1,8$ vs. $7,6 \pm 1,7 ; p=0,026)$, maior tempo diário assistindo televisão e/ou utilizando computador $[180,0$ minutos/dia $(0,0 ; 360,0)$ vs. $120,0$ minutos/dia $(0,0 ; 420,0) ; p=0,018]$, além do relato menos frequente de sudorese durante a prática de exercícios físicos (48,8\% vs. 73,1\%; $p=0,049)$ (Tabela 3). Tais evidências revelam que são as múltiplas variáveis que podem contribuir para a evolução ponderal insatisfatória, sobre as quais será discutido a seguir. 
Tabela 1 - Comparação das variáveis antropométricas de mulheres com excesso de peso na linha de base, conforme evolução ponderal após intervenção. Belo Horizonte, Brasil. 2007-2009.

\begin{tabular}{|c|c|c|c|}
\hline \multirow[b]{2}{*}{ Variáveis } & \multicolumn{2}{|c|}{ Evolução ponderal } & \multirow{2}{*}{$\begin{array}{c}\text { Valor } \\
\text { p }\end{array}$} \\
\hline & $\begin{array}{l}\text { Insatisfatória } \\
(\mathrm{n}=44)\end{array}$ & $\begin{array}{r}\text { Satisfatória } \\
(\mathrm{n}=26)\end{array}$ & \\
\hline $\operatorname{Peso}(\mathbf{k g})^{\dagger}$ & $81,0 \pm 11,7$ & $79,2 \pm 9,7$ & $0,516^{1}$ \\
\hline Índice de Massa Corporal $(\mathrm{kg} / \mathrm{m} 2)^{\S}$ & $\begin{array}{l}33,5 \\
(26,0 ; 42,7)\end{array}$ & $\begin{array}{r}32,9 \\
(29,3 ; 43,0)\end{array}$ & $0,378^{2}$ \\
\hline Circunferência da cintura $(\mathbf{c m})^{\dagger}$ & $97,4 \pm 8,1$ & $96,9 \pm 7,5$ & $0,775^{1}$ \\
\hline Classificação CC (\%) ${ }^{*}$ & & & $0,741^{3}$ \\
\hline Sem risco de complicações associadas à obesidade & 2,3 & 0,0 & \\
\hline Risco elevado de complicações associadas à obesidade & 11,4 & 11,5 & \\
\hline $\begin{array}{l}\text { Risco muito elevado de complicações associadas } \\
\text { à obesidade }\end{array}$ & 86,4 & 88,5 & \\
\hline Razão cintura/quadril (RCQ) ${ }^{\dagger}$ & $0,87 \pm 0,06$ & $0,86 \pm 0,06$ & $0,751^{1}$ \\
\hline Classificação RCQ $(\%)^{* *}$ & & & $0,333^{3}$ \\
\hline Sem risco para o desenvolvimento de doenças & 41,9 & 53,8 & \\
\hline Risco para o desenvolvimento de doenças & 58,1 & 46,2 & \\
\hline
\end{tabular}

Nota: ${ }^{\dagger}$ Média e desvio padrão; ${ }^{\S}$ Mediana, mínimo e máximo; Testes: ${ }^{1}$ Teste t de Student; ${ }^{2}$ Mann Whitney; ${ }^{3}$ Qui Quadrado. *Risco Elevado $\geq 80,0 \mathrm{~cm}$; Risco muito elevado $\geq 88,0 \mathrm{~cm}$; ${ }^{* *}$ Risco para o desenvolvimento de doenças $>0,85$. Fonte: Dados da pesquisa.

Tabela 2 - Comparação dos hábitos e consumo alimentar de mulheres com excesso de peso na linha de base, conforme evolução ponderal após intervenção. Belo Horizonte, Brasil. 2007-2009.

\begin{tabular}{|c|c|c|c|}
\hline \multirow[b]{2}{*}{ Variáveis } & \multicolumn{2}{|c|}{ Evolução ponderal } & \multirow{2}{*}{$\begin{array}{c}\text { Valor } \\
\mathbf{p}\end{array}$} \\
\hline & $\begin{array}{l}\text { Insatisfatória } \\
(\mathrm{n}=44)\end{array}$ & $\begin{array}{r}\text { Satisfatória } \\
(\mathrm{n}=26)\end{array}$ & \\
\hline Número de refeições por dia $\$$ & $4,2(2,0 ; 6,0)$ & $4,0(2,0 ; 6,0)$ & $0,589^{1}$ \\
\hline \multicolumn{4}{|l|}{ Realização do desjejum (\%) } \\
\hline$\geq 4$ vezes & 90,9 & 92,3 & $1,000^{2}$ \\
\hline$<4$ vezes & 9,1 & 7,7 & \\
\hline Beber líquidos durante refeições principais (\%) & 47,7 & 38,5 & \\
\hline \multicolumn{4}{|l|}{ Modo de preparo dos alimentos Carnes (\%) } \\
\hline Cozido/assado & 36,4 & 41,7 & $0,667^{3}$ \\
\hline Refogado/frito & 63,6 & 58,3 & \\
\hline Couve $(\%)$ & & & \\
\hline
\end{tabular}


...continuação - Tabela 2

\begin{tabular}{llll}
\hline Cru & 15,9 & 34,6 & $0,072^{3}$ \\
Refogado & 84,1 & 65,4 &
\end{tabular}

Ovo (\%)

\begin{tabular}{|c|c|c|c|}
\hline Frito & 62,2 & 55,0 & \\
\hline \multicolumn{4}{|l|}{ Consumo diário (\%) } \\
\hline Verduras & 23,3 & 38,5 & $0,177^{3}$ \\
\hline Legumes & 44,2 & 38,5 & $0,641^{3}$ \\
\hline Leite & 46,5 & 61,5 & $0,266^{3}$ \\
\hline Suco artificial & 23,8 & 23,1 & $0,945^{3}$ \\
\hline Banha animal & 4,8 & 11,5 & $0,363^{2}$ \\
\hline Frituras & 26,2 & 15,4 & $0,375^{2}$ \\
\hline \multicolumn{4}{|l|}{ Frutas (\%) } \\
\hline$\geq 4$ vezes/semana & 54,5 & 65,4 & $0,374^{3}$ \\
\hline$<4$ vezes/semana & 45,5 & 34,6 & \\
\hline Per capita diário de sal $(\mathrm{g}){ }^{\S}$ & $5,5(1,7 ; 22,2)$ & $4,6(2,8 ; 25,0)$ & $0,278^{1}$ \\
\hline Per capita diário de açúcar $(\mathrm{g})$ § & $41,7(13,3 ; 166,7)$ & $66,7(25,0 ; 166,7)$ & $0,063^{1}$ \\
\hline Per capita diário de óleo $(\mathrm{mL}){ }^{\S}$ & $20,0(0,0 ; 60,0)$ & $21,2(6,0 ; 45,0)$ & $0,942^{1}$ \\
\hline
\end{tabular}

Nota: ${ }^{\S}$ Mediana, mínimo e máximo; Testes: ${ }^{1}$ Mann Whitney; ${ }^{2}$ Exato de Fisher; ${ }^{3}$ Qui Quadrado. Fonte: Dados da pesquisa.

Tabela 3 - Comparação dos dados sociodemográficos e saúde de mulheres com excesso de peso na linha de base, conforme evolução ponderal após intervenção. Belo Horizonte, Brasil. 2007-2009.

\begin{tabular}{|c|c|c|c|}
\hline \multirow[b]{2}{*}{ Variáveis } & \multicolumn{2}{|c|}{ Evolução ponderal } & \multirow{2}{*}{$\begin{array}{c}\text { Valor } \\
\mathbf{p}\end{array}$} \\
\hline & $\begin{array}{l}\text { Insatisfatória } \\
(\mathrm{n}=44)\end{array}$ & $\begin{array}{r}\text { Satisfatória } \\
(\mathbf{n}=26)\end{array}$ & \\
\hline Idade $\left(\right.$ anos) ${ }^{\dagger}$ & $52,6 \pm 11,9$ & $52,5 \pm 8,7$ & $0,991^{1}$ \\
\hline \multicolumn{4}{|l|}{ Classificação da idade (\%) } \\
\hline Adultas & 61,4 & 73,1 & $0,318^{2}$ \\
\hline Idosas & 38,6 & 26,9 & \\
\hline \multicolumn{4}{|l|}{ Ocupação profissional (\%) } \\
\hline Do lar & 52,3 & 61,5 & $0,451^{2}$ \\
\hline Outras* & 47,7 & 38,5 & \\
\hline
\end{tabular}




\begin{tabular}{|c|c|c|c|}
\hline Renda per capita mensal $(\$)^{\S}$ & $\begin{array}{l}267,00 \\
(167,7 ; 1.790,95) \\
\end{array}$ & $\begin{array}{r}645,00 \\
(255,85 ; 1.881,25) \\
\end{array}$ & $0,491^{3}$ \\
\hline Escolaridade (anos) $)^{\S}$ & $5,5(0,0 ; 13,0)$ & $4,5(0,0 ; 13,0)$ & $0,849^{3}$ \\
\hline \multicolumn{4}{|l|}{ Morbidade referida (\%) } \\
\hline Hipertensão arterial & 60,5 & 34,6 & $0,037^{2}$ \\
\hline Hipercolesterolemia & 27,0 & 46,2 & $0,117^{2}$ \\
\hline Diabetes mellitus & 16,3 & 7,7 & $0,466^{4}$ \\
\hline Hipertrigliceridemia & 13,9 & 5,0 & $0,405^{4}$ \\
\hline Uso de medicamentos (\%) & 86,4 & 69,2 & \\
\hline Horas de sono por noite $^{\dagger}$ & $6,6 \pm 1,8$ & $7,6 \pm 1,7$ & \\
\hline \multicolumn{4}{|l|}{ Autopercepção da saúde (\%) } \\
\hline Muito boa/Boa & 47,7 & 56,0 & $0,509^{2}$ \\
\hline Razoável/ Ruim/Muito ruim & 52,3 & 44,0 & \\
\hline Prática regular de atividade física (\%) & 93,2 & 96,2 & $1,000^{4}$ \\
\hline Televisão/computador por dia (minutos) ${ }^{\S}$ & $180,0(0,0 ; 360,0)$ & $120,0(0,0 ; 420,0)$ & $0,018^{3}$ \\
\hline Sudorese ao realizar exercícios físicos (\%) & 48,8 & 73,1 & $0,049^{2}$ \\
\hline \multicolumn{4}{|l|}{ Autopercepção corporal (\%) } \\
\hline Normal & 0,0 & 3,8 & $0,377^{4}$ \\
\hline Gorda & 100,0 & 96,2 & \\
\hline \multicolumn{4}{|l|}{ Satisfação corporal (\%) } \\
\hline Satisfeita & 39,5 & 44,0 & $0,718^{2}$ \\
\hline Insatisfeita & 60,5 & 56,0 & \\
\hline Tentativa de emagrecer (últimos 6 meses-\%) & 69,8 & 53,8 & $0,182^{2}$ \\
\hline
\end{tabular}

Nota: ${ }^{\dagger}$ Média e desvio padrão; ${ }^{\circledR}$ Mediana, mínimo e máximo - Média do salario mínimo no período (2007-2009) $=\mathrm{R} \$ 420,00$; Testes: ${ }^{1}$ Teste $t$ de Student; ${ }^{2}$ Qui Quadrado; ${ }^{3}$ Mann Whitney; ${ }^{4}$ Exato de Fisher; ${ }^{*}$ Outras: Aposentada, autônoma, desempregada, empregada doméstica e outras profissões. Fonte: Dados da pesquisa.

\section{DISCUSSÃO}

Identificou-se prevalência significativa de evolução ponderal insatisfatória (redução do peso corporal inferior a 5\%) entre mulheres com excesso de peso que participaram de acompanhamento nutricional na APS, sendo esta condição associada, na linha de base, à presença de HA, pior hábito de sono, sedentarismo e provável menor intensidade da prática de exercício físico.

A evolução ponderal insatisfatória foi averiguada entre $62,9 \%$ das participantes, percentual semelhante ao observado em estudo realizado no interior do estado de São Paulo com adultos com excesso de peso após 10 semanas de intervenção nutricional, psicológica e física em grupo $(62,1 \%)^{16}$. Prevalência inferior foi verificada em intervenção individual sobre alimentação e atividade física conduzida no Rio de Janeiro-RJ, com duração de quatro meses $(55,3 \%)^{5}$, e em intervenção individual 
desenvolvida com adultos norte-americanos com excesso de peso e intolerância à glicose avaliados após um ano de acompanhamento $(57,0 \%)^{17}$.

Em contrapartida, outro trabalho realizado no interior de São Paulo, no qual adultos nipobrasileiros com excesso de peso e/ou obesidade abdominal participaram de um atendimento individual e uma dinâmica de grupo, obteve prevalência de evolução ponderal insatisfatória superior à frequência observada no presente estudo $(92,0 \%)^{18}$. Semelhantemente, na Holanda, estudo de intervenção conduzido durante seis meses com adultos e idosos com excesso de peso, observou-se evolução insatisfatória do peso corporal entre 80,0\% dos indivíduos que participaram somente de intervenção nutricional individual e entre 68,0\% daqueles que receberam, adicionalmente, orientações sobre atividade física ${ }^{19}$. No entanto, a comparação dos resultados deste estudo com a literatura deve ser realizada com cautela, tendo em vista as diferenças metodológicas verificadas, como maior ou menor tempo de acompanhamento e o delineamento das intervenções.

Neste trabalho, maior prevalência de evolução ponderal insatisfatória foi identificada entre as mulheres que relataram possuir HA. A relação entre a evolução ponderal e a presença de comorbidades constitui uma questão ainda contraditória. Por um lado, a presença de DCNT poderia contribuir para a evolução satisfatória do peso em função da possibilidade de o tempo de convivência com a doença favorecer a melhor compreensão sobre seu quadro de saúde e oportunizar maior adesão às orientações.

Por outro lado, apresentar doenças crônicas poderia dificultar a evolução e adesão às propostas de intervenção nutricional, condição que pode se associar a diversos fatores, como a ausência de apoio familiar, ressignificação das práticas alimentares e limitado senso de necessidade em relação ao cuidado requerido pela doença $\mathrm{a}^{4,5,7,9}$.

Apesar da presença de HA exigir cuidados permanentes, com modificações no estilo de vida, incluindo controle do peso, adoção de uma alimentação equilibrada e prática regular de exercícios físicos, verifica-se, de maneira geral, uma baixa adesão a estes cuidados. Acredita-se que o não reconhecimento da importância do controle do peso corporal para o seu tratamento, restringindo, possivelmente, as orientações à redução do consumo de sal e alimentos ricos em sódio, possa ser um contribuinte para este panorama ${ }^{20}$.

Os resultados desse estudo indicam também a importância de outros hábitos de vida na predição da evolução ponderal insatisfatória, como o menor tempo de sono, o maior tempo gasto assistindo televisão ou utilizando computador e a ausência de sudorese durante a prática de exercícios físicos.

A restrição do sono pode se relacionar ao aumento do IMC devido a alterações endócrinas que favorecem o aumento do apetite e da ingestão alimentar, além de uma possível influência no decréscimo da prática diária de exercícios físicos, atuando assim, de forma sinérgica para uma possível evolução ponderal insatisfatória ${ }^{21}$.

Adicionalmente, o tempo gasto assistindo televisão ou utilizando computador constitui um dos principais comportamentos sedentários atuais que oportunizam o balanço energético positivo e consequente incremento do peso $^{22}$. Paralelamente, verificou-se uma menor sudorese durante a prática de exercícios físicos entre aqueles que evoluíram insatisfatoriamente. A prática de exercícios físicos aumenta a produção de suor, que varia proporcionalmente com a intensidade do treinamento, além de outros fatores $^{23}$.

Dessa forma, o relato menos frequente de sudorese pelas participantes que não evoluíram de forma satisfatória pode ser sugestivo de que a frequência e ou intensidade das atividades realizadas eram insuficientes para promover o gasto energético necessário para a redução do peso, reforçando a importância da adequação do exercício físico associado à alimentação equilibrada como estratégia favorecedora da evolução ponderal satisfatória ${ }^{23}$.

Este estudo se destaca por buscar identificar fatores preditores da evolução ponderal insatisfatória após participação em intervenção realizada no contexto dos serviços de saúde, cenário de um número limitado de estudos de intervenção ${ }^{24}$. Destaca-se ainda que a pesquisa prezou pela garantia da validade 
interna, evidenciada pelo desenvolvimento da intervenção com base em evidências científicas, pelo rigor na coleta de dados, realização de treinamentos periódicos, acompanhamento contínuo da intervenção pelo pesquisador e definição criteriosa da variável de desfecho. Ademais, a integração entre os serviços de saúde da APS, Programa Academia da Saúde e UBS, para a oferta do cuidado às usuárias, possibilitou o manejo diferenciado do excesso de peso. Contudo, este se mostrou ainda insuficiente para gerar intervenções exitosas para a maior parte do grupo estudado, haja vista a elevada prevalência de evolução insatisfatória do peso.

Considera-se que não foi possível avaliar outros possíveis fatores intervenientes na evolução ponderal relacionados ao percurso da intervenção, como o apoio familiar, motivação para o tratamento, adesão às orientações nutricionais, exposição à intervenção e sua repercussão, reconhecidamente elementos passíveis de influenciar o sucesso das ações ${ }^{4}$. Entretanto, é válido realçar que a avaliação destes fatores não consistiu o objetivo do estudo, uma vez que, se julgou importante identificar as características das mulheres com excesso de peso antes da intervenção associadas à evolução ponderal, de modo a auxiliar no ajustamento das ações e contribuir para o planejamento de novas investigações.

Os resultados apontam a relevância de ampliar a abordagem terapêutica do excesso de peso para além da promoção da alimentação adequada e saudável, abarcando outros fatores, como a presença de comorbidades, tempo de sono, prática e intensidade de exercícios físicos, e hábitos sedentários com vistas a favorecer a estruturação de ações que atendam às necessidades específicas dos participantes e assim, contribuir para o maior êxito nas intervenções.

Neste aspecto, esses achados são congruentes com as diretrizes contidas na Linha de Cuidado para Pessoas com Excesso de Peso, desenvolvida pelo Ministério da Saúde brasileiro, que apontam a interdisciplinaridade das ações ofertadas aos indivíduos adultos com excesso de peso e comorbidades como ação prioritária visando à maior efetividade no tratamento destes indivíduos ${ }^{25}$.
Por fim, acredita-se que os achados aqui obtidos possam contribuir para ampliar a discussão sobre como atingir melhores resultados mediante intervenções nutricionais individuais na APS. Acreditamos que melhores taxas de evolução ponderal satisfatória poderão ser obtidas mediante a atuação conjunta de outros profissionais de saúde, além do nutricionista, em diferentes momentos de oferta do cuidado na APS. Isso, tendo em vista que ações interdisciplinares, disponibilizadas em rede, poderão favorecer a oferta qualificada do cuidado aos indivíduos com excesso de peso e comorbidades.

Além disso, reitera-se ser fundamental que as intervenções sejam planejadas de forma a incentivar a participação ativa dos indivíduos nas decisões sobre a sua saúde, favorecendo o autocuidado apoiado e a corresponsabilização pelo alcance das metas do plano terapêutico, contribuindo para que o usuário se torne e se reconheça como protagonista do seu cuidado, apoiado pela rede de profissionais que o acompanha longitudinalmente.

\section{CONCLUSÃO}

Verificou-se significativo percentual de mulheres com evolução ponderal insatisfatória. Considerando as variáveis do início do acompanhamento, esta condição se associou positivamente à presença de $\mathrm{HA}$, hábito de dormir menos horas por noite, maior tempo diário assistindo televisão e/ou utilizando computador, além do relato menos frequente de sudorese durante a prática de exercícios físicos. Estes achados sugerem a necessidade de intervenções terapêuticas diferenciadas, ampliadas, que extrapolem a alimentação, de modo a considerar a complexidade envolvida no manejo do excesso de peso almejando o maior sucesso das ações, conforme preconizado pela Linha de Cuidado para Pessoas com Excesso de Peso, proposta pelo Ministério da Saúde.

À luz dessa complexidade, destacamos a importância, além da interdisciplinaridade, da construção de vínculo entre profissionais e usuários, e da individualização das condutas de acordo com as necessidades do indivíduo, 
especialmente considerando o seu perfil socioeconômico, de saúde e de nutrição.

A busca por melhores taxas de evolução ponderal nas ações é uma questão desafiadora, sendo o conhecimento sobre os limitantes para o alcance de metas fundamental para o desenvolvimento de estratégias mais efetivas e menos onerosas para o sistema de saúde.

FINANCIAMENTO: Fundação de Amparo à Pesquisa de Minas Gerais (EDT-3245/06).

COLABORAÇÃO: Mariana Souza Lopes (MSL) - coleta, análise e interpretação dos dados, e redação do manuscrito. Maria Júlia Gonçalves Barbosa (MJGB) - análise einterpretação dos dados, e redação do manuscrito. Nathália Luiza Ferreira (NLF)- coleta, análise, e interpretação dos dados, revisão crítica e intelectual do conteúdo. Luana Caroline dos Santos (LCS) - revisão crítica e intelectual do conteúdo. Aline Cristine Souza Lopes (ACSL) - concepção e delineamento do estudo, aquisição de dados, obtenção do financiamento, análise e interpretação dos dados, revisão crítica e intelectual do conteúdo. Todos os autores aprovaram a versão a ser publicada.

\section{REFERÊNCIAS}

1. Chan RSM, Woo J. Prevention of overweight and obesity: how effective is the current public health approach. Int J Environ Res Public Health. 2010; 7(3):765-83.

2. Rodrigues EM, Piza FPT, Boog MCF. Resgate do conceito de aconselhamento no contexto do atendimento nutricional. Rev Nutr. $2005 ; 18(1): 119-28$.

3. Institute of Medicine (IOM). Committee to develop criteria for evaluating the outcomes of approaches to prevent and treat obesity. Weighing the options: criteria for evaluating weight-management programs. Obes Res. 1995; 3:591-604.

4. Guimarães NG, Dutra ES, ItO MK, Carvalho KMB. Adesão a um programa de aconselhamento nutricional para adultos com excesso de peso e comorbidades. Rev Nutr. 2010; 23(3):323-33.

5. Barbato KBG, Martins RCV, Rodrigues MLG, Braga JU, Francischetti EA, Genelhu V. Efeitos da redução de peso superior a 5\% nos perfis hemodinâmico, metabólico e neuroendócrino de obesos grau I. Arq Bras Cardiol. 2006; 87(1):12-20.

6. Souza JMB; Castro MM; Maia EMC, Ribeiro DAN; Almondes KM; Silva NG. Obesity and treatment: behavioral and social challenge. Rev Bras Ter Cogn. 2005; 1(1):59-67.

7. Vieira CM, Cordeiro SN, Magdaleno JR, Turato ER. Significados da dieta e mudanças de hábitos para portadores de doenças metabólicas crônicas: uma revisão. Ciênc Saúde Coletiva. 2011; 16(7):3161-8.

8. Bautista-Castaño I, Molina-Cabrillana J, Montoya-Alonso JA, Serra-Majem L. Variables predictive of adherence to diet and physical activity recommendations in the treatment of obesity and overweight, in a group of spanish subjects. Int J Obes Relat Metab Disord. 2004; 28(5):697-705.

9. Dosse C, Cesarino CB, Martin JFV, Castedo MCA. Fatores associados à não adesão dos pacientes ao tratamento de hipertensão arterial. Rev Latino-am Enfermagem. 2009; 17(2):201-6.

10. Mendonça RD, Mingoti SA, Jaime PC, Lopes ACS. Impacto de intervenção nutricional sobre o perfil alimentar e antropométrico de usuárias do Programa Academia da Saúde. Ciênc Saúde Coletiva (Impresso). 2015; 20(6):1937-46.

11. Lopes ACS, Ferreira AD, Santos LC. Atendimento nutricional na atenção primária à saúde: proposição de protocolos. Nutrição em Pauta. 2010; 101:40-4.

12. World Health Organization (WHO). Obesity: preventing and managing the global epidemic. report of a who consultation on obesity. Geneva: WHO; 1998. 265p.

13. Nutrition Screening Initiative - NSI. Nutrition interventions manual for professionals caring for older Americans. Washington: The Nutrition Screening Initiative, 1992. 130p.

14. World Health Organization (WHO). Waist circumference and waist-hip ratio: report of a who expert consultation, Geneva, 8-11 December 2008. Geneva, 2011. 48p.

15. Ferreira NL, Mingoti SA, Jaime PC, Lopes ACS. Effectiveness of nutritional intervention in overweight women in primary health care. Rev Nutr. 2014; 27(6):677-87.

16. Bueno JM, Leal FS, Saquy LPL, Santos CB, Ribeiro RPP. Educação alimentar na obesidade: adesão e resultados antropométricos. Rev Nutr. 2011; 24(4):575-84.

17. Tuomilehto J, Lindström J, Eriksson JG, Valle TT, Hämäläinen H, llanne-Parikka P, et al. Prevention of type 2 diabetes mellitus by changes in lifestyle among subjects with impaired glucose tolerance. N Engl J Med. 2001; 344(18):1343-50.

18. Camargo MAG, Franco LJ, Gimeno SGA, Ferreira SRG, Sartorelli DS. Fatores preditores do alcance das metas de um programa de intervenção em nipo-brasileiros. Cad Saúde Pública. 2010; 26(6):1141-52.

19. Molenaar EA, Van Ameijden EJ, Vergouwe Y, Grobbee DE, Numans ME. Effect of nutritional counselling and nutritional plus exercise counselling in overweight adults: a randomized trial in multidisciplinary primary care practice. Fam Pract. 2010; 27(2):143-50. 20. Figueiredo NN, Asakura L. Adesão ao tratamento anti-hipertensivo: dificuldades relatadas por indivíduos hipertensos. Acta Paul Enferm. 2010; 23(6):782-7.

21. Crispim CA, Zalcman I, Dáttilo M, Padilha HG, Tufik S, Mello, MT. Relação entre sono e obesidade: uma revisão da literatura. Arq Bras Endocrinol Metab. 2007; 51(7):1041-9. 
22. Inoue S, Sugiyama T, Takamiya T, Oka K, Owen N, Shimomitsu T. Television viewing time is associated with overweight/obesity among older adults, independent of meeting physical activity and health guidelines. J Epidemiol. 2012; 22(1):50-6.

23. Carvalho T, Mara LS. Hidratação e nutrição no esporte. Rev Bras Med Esporte. 2010; 16 (2):145-8.

24. Canella DS, Silva ACF, Jaime PC. Produção científica sobre nutrição no âmbito da Atenção Primária à Saúde no Brasil: uma revisão de literatura. Ciênc Saúde Coletiva. 2013; 18(2):297-308.

25.Brasil. Ministério da Saúde. Estabelece regulamento técnico, normas e critérios para a assistência de alta complexidade ao indivíduo com obesidade. Portaria n. 425, de 19 de março de 2013. Diário Oficial da União. 20 de março, 2013., Secção I. 